\title{
O069. Menstrual cycle affects cortical excitability differently in females with migraine and in healthy controls: a new perspective by cross modal sound induced flash illusions
}

Simona Maccora ${ }^{1 *}$, Carlo Mannina ${ }^{1}$, Nadia Bolognini ${ }^{2,3}$, Piera Paladino ${ }^{1}$, Roberta Baschi ${ }^{1}$, Giuseppe Cosentino ${ }^{1}$, Brigida Fierro ${ }^{1}$, Giuseppe Vallar ${ }^{2,3}$, Filippo Brighina ${ }^{1}$

From Abstracts from the 1st Joint ANIRCEF-SISC Congress

Rome, Italy. 29-31 October 2015

\section{Introduction}

The sound-induced flash illusions (SIFI) represent a valid tool to explore multimodal perception and are critically dependent on visual and acoustic cortical excitability [1,2]. In a previous study [3], we observed a significant reduction of illusions in migraine patients with respect to healthy controls, probably due to a condition of visual cortex hyperexcitability. Aim of the present study was to evaluate SIFI perceptions in healthy women and patients with menstrual migraine and to describe the effects of cyclical change of steroid hormones and cortical responsiveness.

\section{Materials and methods}

Nineteen women (11 affected with menstrual migraine, 8 healthy controls) were enrolled. Serum determination for sexual hormones (estradiol, progesterone) and a SIFI trial were performed in all participants in two different sessions on the 14th and 27th day of menstrual cycle.

\section{Results}

Healthy women showed more illusions in the premenstrual (27th day) than in the luteal phase (14. day) $(\mathrm{p}<0.01)$. Migraine patients did not show any difference during the two phases of menstrual cycle; they saw significantly less fissions illusions $(\mathrm{p}<0.001)$ with respect to healthy women at 27 th, but not at 14 th day of menstrual cycle.

\footnotetext{
* Correspondence: simonamaccora1@gmail.com

${ }^{1}$ Dipartimento di Biomedicine Sperimentali e Neuroscienze Cliniche (BioNeC), Università di Palermo, Palermo, Italy

Full list of author information is available at the end of the article
}

\section{Conclusions}

Results in healthy subjects are in line with hormonal effects on cortical excitability. During late follicular phase, the increase of estradiol could determine visual cortex hyperexcitability corresponding to a reduction of SIFI. Conversely, premenstrual fall of estradiol would account for restored illusions. Persistence of a reduced illusory susceptibility in both phases of menstrual cycle in migraine patients would underlie a reduced responsivity of visual cortex to hormonal fluctuation.

Written informed consent to publish was obtained from the patient(s).

\section{Authors' details \\ ${ }^{1}$ Dipartimento di Biomedicine Sperimentali e Neuroscienze Cliniche (BioNeC), Università di Palermo, Palermo, Italy. ${ }^{2}$ Dipartimento di Psicologia, Università di Milano-Bicocca, Milan, Italy. ${ }^{3}$ Laboratorio di Neuropsicologia, IRCCS Istituto Auxologico Italiano, Milan, Italy. \\ Published: 28 September 2015 \\ References \\ 1. Shams L, Kamitani Y, Shimojo S: Illusions. What you see is what you hear. Nature 2000, 408(408):788. \\ 2. Bolognini N, Rossetti A, Casati C, Mancini F, Vallar G: Neuromodulation of multisensory perception: a tDCS study of the sound-induced flash illusion. Neuropsychologia 2011, 49(2):231-237. \\ 3. Brighina F, Bolognini N, Cosentino G, Maccora S, Paladino P, Baschi R, Vallar G, Fierro B: Visual cortex hyperexcitability in migraine by sound- induced flash illusions. Neurology 2015, 84(20):2057-2061.}

doi:10.1186/1129-2377-16-S1-A141

Cite this article as: Maccora et al:: O069. Menstrual cycle affects cortical excitability differently in females with migraine and in healthy controls: a new perspective by cross modal sound induced flash illusions. The Journal of Headache and Pain 2015 16(Suppl 1):A141. 